\title{
Integrated market selection and production planning: complexity and solution approaches
}

\author{
Wilco Van den Heuvel • O. Erhun Kundakcioglu • \\ Joseph Geunes • H. Edwin Romeijn • \\ Thomas C. Sharkey • Albert P. M. Wagelmans
}

Received: 18 April 2010 / Accepted: 5 January 2011 / Published online: 24 February 2011

(C) The Author(s) 2011. This article is published with open access at Springerlink.com

\begin{abstract}
Emphasis on effective demand management is becoming increasingly recognized as an important factor in operations performance. Operations models that account for supply costs and constraints as well as a supplier's ability to influence demand characteristics can lead to an improved match between supply and demand. This paper presents a class of optimization models that allow a supplier to select, from a set of potential markets, those markets that provide maximum profit when
\end{abstract}

W. Van den Heuvel $(\varangle)$. A. P. M. Wagelmans Econometric Institute, Erasmus University Rotterdam, P.O. Box 1738, 3000 DR Rotterdam, The Netherlands e-mail: wvandenheuvel@ese.eur.nl

A. P. M. Wagelmans

e-mail: wagelmans@ese.eur.nl

O. E. Kundakcioglu

Department of Industrial Engineering, University of Houston, E209 Engineering Bldg. 2, Houston, TX 77204-4008, USA

e-mail: erhun@uh.edu

J. Geunes

Department of Industrial and Systems Engineering,

University of Florida, P.O. Box 116595, Gainesville, FL 32611-6595, USA

e-mail: geunes@ise.ufl.edu

H. E. Romeijn

Department of Industrial and Operations Engineering,

The University of Michigan, 1205 Beal Avenue, Ann Arbor, MI 48109-2117, USA

e-mail: romeijn@umich.edu

T. C. Sharkey

Center for Industrial Innovation, Rensselaer Polytechnic Institute,

110 8th St. Suite 5015, Troy, NY 12180-3590, USA

e-mail: sharkt@rpi.edu 
production/procurement economies of scale exist in the supply process. The resulting optimization problem we study possesses an interesting structure and we show that although the general problem is $\mathcal{N} \mathcal{P}$-complete, a number of relevant and practical special cases can be solved in polynomial time. We also provide a computationally very efficient and intuitively attractive heuristic solution procedure that performs extremely well on a large number of test instances.

Keywords Lot-sizing $\cdot$ Market selection $\cdot$ Complexity

Mathematics Subject Classification (2000) $68 \mathrm{Q} 25 \cdot 90 \mathrm{~B} 30 \cdot 90 \mathrm{C} 39 \cdot 90 \mathrm{C} 59$

\section{Introduction}

Production/procurement planning problems are among the most well studied problems in the operations literature. The majority of research in this area focuses on managing the supply side of the problem when capacities and/or nonlinear cost structures lead to problems containing a combinatorial structure. Much of this past research provides efficient solution methods for minimizing the supply cost incurred in order to meet forecasted demand. Recent research recognizes the importance of accounting for the levers suppliers have for managing or shaping demand in order to ensure the most profitable match between supply and demand (see, e.g., [21] and [9]). Spurred on by the development of research in the area of revenue management in the past 20 years (see [24] for a thorough discussion of this field), a number of recent papers have considered the pricing lever that suppliers can use to help shape demand within procurement planning contexts (see, e.g., $[2-5,7,13,15,16,29]$ ). We note that these more recent works built on the foundations provided by [11,26,27,31], and [20]. For a review on coordinating pricing and production/procurement decisions we refer to [33].

While pricing can be viewed as a (partial) determinant of the demands a supplier implicitly selects for a product, a supplier must often first make an explicit selection of the set of markets or sales channels in which it will offer the item. The model we consider focuses on this higher level demand shaping decision, and its role in determining the best match between supply and demand when the production/procurement process involves economies of scale in output. The class of problems we consider can be stated simply and concisely: Given a set of potential markets (or customers) for an item and the sales (and revenue) levels implied by each market in every period over a finite horizon, determine a subset of markets that maximizes net profit, given that all demand for each selected market must be satisfied. Clearly, when supply costs are linear, each market's profitability can be determined in isolation, and the resulting problem is easily solved. However, economies of scale in production/procurement for an item can lead to a high degree of combinatorial complexity in determining a subset of markets that maximizes a supplier's net profit from sales. In particular, we will use a supply cost structure that is consistent with the well-known Wagner-Whitin problem [32]. 
The resulting integrated market selection and production planning problem (which we will refer to as the market selection problem (MSP)) was first proposed in [14], while [22] developed a constant-factor polynomial-time approximation algorithm for a related class of market rejection problems in cost-minimization form.

Despite this prior work, this problem's complexity has to date remained open. In this paper, we will not only show that the MSP is $\mathcal{N} \mathcal{P}$-hard, but also that no constant-factor polynomial-time approximation algorithm exists (unless $\mathcal{P}=\mathcal{N} \mathcal{P}$ ).

The MSP has a particularly interesting structure, as a number of its practical special cases are polynomially solvable. For instance, given a set of $M$ markets that must be served, then the resulting problem is equivalent to a standard uncapacitated lot sizing problem, which can be solved in $\mathcal{O}(T \log T)$ in general (where $T$ is the planning horizon length) and $\mathcal{O}(T)$ under time-invariant costs (see, e.g., [30]). On the other hand, given a prescribed set of procurement periods, it is straightforward to determine, for each market, whether the average revenue per unit of demand exceeds the minimum average cost per unit (which, after a preprocessing step in which these average costs per demand unit are determined in $\mathcal{O}(M T)$ time, can be done in $\mathcal{O}(M)$ time, where $M$ is the number of potential markets).

Despite the fact that MSP is $\mathcal{N} \mathcal{P}$-hard in general, we will explore a number of special cases of the problem that may occur in practice and can be solved in polynomial time. Moreover, we provide a new heuristic solution approach for the problem and demonstrate the effectiveness of this approach on a large number of test instances. Thus, the contributions of this paper include (i) characterization of the worst-case complexity of an interesting and relevant problem class; (ii) identification of a number of polynomially solvable special cases; and (iii) providing an intuitively attractive heuristic solution method for the problem with excellent performance results.

The remainder of this paper is organized as follows. Section 2 formally defines and formulates the integrated market selection and procurement planning problem. Section 3 demonstrates the $\mathcal{N} \mathcal{P}$-hardness of this problem and shows that there does not exist a polynomial-time approximation algorithm that guarantees a solution within some given percentage of the optimal profit unless $\mathcal{P}=\mathcal{N} \mathcal{P}$. Furthermore, it shows the relation between the MSP and a class of games that has recently been proposed in the literature: economic lot-sizing games. Section 4 provides polynomial-time solution approaches for a number of special cases of the problem. In Sect. 5 we propose a new heuristic and present a heuristic previously proposed in the literature. In Sect. 6 we perform a computational study which shows that the heuristic works very well. We provide brief concluding remarks and directions for future work in Sect. 7.

\section{Formal problem description}

The MSP can be described as follows. We are given $M$ potential markets and a revenue $R_{m}$ associated with market $m(m=1, \ldots, M)$. Market $m$ has a known demand $d_{t}^{m}$ in each period $t$ of a discrete and finite time horizon of length $T$. A supplier may choose to either satisfy the demand of a market or to reject a market. If the supplier selects a market, the market's demand must be satisfied in all periods. If the supplier rejects a market, then none of the market's demand is satisfied and the revenue is lost. 
Given a selection of markets, the supplier faces a production planning problem. The cost of a production plan consists of a fixed setup cost $K_{t}$ in period $t$ if and only if production is positive in period $t$, a production cost $p_{t}$ for each unit produced in period $t$, and a holding cost of $h_{t}$ for each item in inventory at the end of period $t$, for $t=1, \ldots, T$. The objective of the manufacturer is to maximize total profit, i.e., to select a set of markets that maximizes revenues less the production and holding costs associated with satisfying the selected market demands. For convenience, we define $c_{i, t}^{m}=d_{t}^{m}\left(p_{i}+\sum_{j=i}^{t-1} h_{j}\right)$ as the cost of satisfying market $m$ demand in period $t$ using production in period $i$, and let $x_{i, t}^{m}$ denote the decision variable for the proportion of demand $d_{t}^{m}$ satisfied using production in period $i(i, t=1, \ldots, T, i \leq t$; $m=1, \ldots, M)$. We define the binary variables $y_{i}=1$ if there is a setup in period $i$ ( 0 otherwise) for $i=1, \ldots, T$, and $z_{m}=1$ if market $m$ is selected ( 0 otherwise) for $m=1, \ldots, M$.

Then the MSP can be formulated as the following mixed integer program (MIP):

$$
\begin{array}{cl}
\max \sum_{m=1}^{M} R_{m} z_{m}- & \sum_{t=1}^{T}\left(K_{t} y_{t}+\sum_{i=1}^{t} \sum_{m=1}^{M} c_{i, t}^{m} x_{i, t}^{m}\right) \\
\text { s.t. } \sum_{i=1}^{t} x_{i, t}^{m}=z_{m}, & t=1, \ldots, T, m=1, \ldots, M, \\
x_{i, t}^{m} \leq y_{i}, & i, t=1, \ldots, T, i \leq t ; m=1, \ldots, M, \\
x_{i, t}^{m} \geq 0, & i, t=1, \ldots, T, i \leq t ; m=1, \ldots, M, \\
y_{i} \in\{0,1\}, & i=1, \ldots, T, \\
z_{m} \in\{0,1\}, & m=1, \ldots, M .
\end{array}
$$

The objective function maximizes total revenue minus total production cost. The first set of constraints ensures that demand is satisfied when a market is selected. Constraints (1c) are setup forcing constraints: production in period $i$ can only occur if there is a setup in that period. Constraints (1d) ensure that production is nonnegative, while constraints (1e) and (1f) represent the binary setup and market selection decisions.

For a given selection of markets or a market selection for short, i.e., a vector $z \in$ $\{0,1\}^{M}$, the problem boils down to a classical lot-sizing problem [32], where demand in a period equals the sum of the demands of the selected markets in that period. Federgruen and Tzur [10], Wagelmans et al. [30], and Aggarwal and Park [1] show that the classical lot-sizing problem can be solved in $\mathcal{O}(T \log T)$ time in the case of general cost parameters and in $\mathcal{O}(T)$ time in the case of non-speculative motives, i.e., when $p_{t}+h_{t} \geq p_{t+1}$ for $t=1, \ldots, T-1$. The polynomial solvability of the MSP for a given market selection is also captured in (1). Namely, for a given market selection the associated subproblem of formulation (1) is equivalent to the so-called plant location formulation. Krarup and Bilde [19] show that the linear relaxation of this formulation has integer $y$-variables in an optimal solution. This then, in turn, implies that in the presence of the binary constraints (1f), the binary constraints (1e) can be relaxed.

On the other hand, given any production plan (i.e., given the $y$-variables), the problem is also easy to solve. As the zero-inventory property holds, i.e., production only occurs in a period with no incoming inventory, one can calculate the variable 
production and holding cost for every market. Then, only markets for which the revenues exceed the variable production costs are selected. Formally, one can show that for fixed $y$-variables, the problem decomposes in $M$ subproblems and the coefficient matrix of every subproblem is totally unimodular. This implies in addition that, in the presence of the binary constraints (1e), the binary constraints (1f) can be relaxed. Thus, the problem is easy to solve if either a selection of markets (the $z$-variables) or a production plan (the $y$-variables) is fixed. Moreover, one of the constraint sets (1e) and (1f) can be relaxed. Unfortunately, however, we cannot relax both constraint sets simultaneously, so the problem is not easily solvable in general.

\section{Complexity results}

In this section we will show that the market selection problem is strongly $\mathcal{N} \mathcal{P}$-hard. Proving this result requires the following steps:

1. We first construct a special case of the MSP in which costs and revenues have a special structure and where, in each period, at most one market has positive demand.

2. We then show that, for this special case, a solution with positive profit exists if and only if a selection of markets exists with the property that all maximal nonempty subsequences of consecutive periods with positive demand are of even length. We will call this the even subsequence property.

3. Finally, we will show that the problem of identifying a set of markets satisfying the even subsequence property is $\mathcal{N} \mathcal{P}$-complete through a reduction from the $3 \mathrm{SAT}$ problem.

After showing that the MSP is strongly $\mathcal{N} \mathcal{P}$-hard, we will end the section with a discussion of approximation results and show the implication of our complexity result for a class of cooperative games.

\subsection{A special case of the MSP}

The class of instances of the MSP that we employ is based on an instance of the uncapacitated lot-sizing problem with $d_{t}=1, K_{t}=2, h_{t}=1$, and $p_{t}=0$ for $t=1, \ldots, T$.

When the horizon $T$ is infinite, it is optimal to produce 2 units in every odd period, as this minimizes the average cost per time period. Define a subplan as an interval for which demands are satisfied by the same production period. Then the optimality property implies that every subplan in an optimal solution consists of 2 periods, and the average cost per demand unit equals $\frac{3}{2}$. It is easy to see that the same solution structure yields an optimal solution when $T$ is finite and even, in which case the optimal cost is given by $C(T)=\frac{3}{2} T$ and the average cost per demand unit by $\bar{C}(T)=\frac{3}{2}$.

Now consider a problem instance with a finite horizon $T$ where $T=2 n+1$ for some $n \in \mathbb{N}$, i.e., $T$ is odd. An optimal solution for this instance consists of $n$ two-period subplans and 1 subplan that supplies demand in a single period. 
The cost of this solution is

$$
C(T)=\frac{3}{2} T+\frac{1}{2}
$$

(Note that a solution with $n-1$ subplans consisting of two periods and 1 subplan consisting of 3 periods has the same cost.) The average cost per unit of demand for this instance equals

$$
\bar{C}(T)=\frac{3}{2}+\frac{1}{2 T}>\frac{3}{2} .
$$

Based on this lot-sizing problem, we define the following class of instances of the MSP with markets $\mathcal{M}=\{1, \ldots, M\}$ :

- the planning horizon $T$ is odd;

- $K_{t}=2, h_{t}=1, p_{t}=0$ for $t=1, \ldots, T$;

- in each period $t$ there is exactly one market $m \in \mathcal{M}$ with $d_{t}^{m}=1$, and $d_{t}^{m^{\prime}}=0$ for all $m^{\prime} \neq m$

- $R_{m}=r \sum_{t=1}^{T} d_{t}^{m}$ with $r=\bar{C}(T)$.

For this class of problem instances, we will next show that an optimal solution exists containing positive profit if and only if a selection of markets exists that satisfies the even subsequence property, i.e., the property that all subsequences of consecutive periods with positive demand are of even length. Now let $\mathcal{M}^{\prime} \subset \mathcal{M}$ denote a set of selected markets and $\Pi\left(\mathcal{M}^{\prime}\right)$ the corresponding profit. Let $d^{\mathcal{M}^{\prime}}$ be the demand sequence induced by $\mathcal{M}^{\prime}$, i.e., $d_{t}^{\mathcal{M}^{\prime}}=\sum_{m \in \mathcal{M}^{\prime}} d_{t}^{m}$. Then a market selection $\mathcal{M}^{\prime}$ satisfies the even subsequence property if, for any $s<t$ with $d_{s}^{\mathcal{M}^{\prime}}=d_{t+1}^{\mathcal{M}^{\prime}}=0$ and $d_{i}^{\mathcal{M}^{\prime}}=1$ for $i=s+1, \ldots, t$ we have that $t-s$ is even (where, for convenience, we let $\left.d_{0}^{\mathcal{M}^{\prime}}=d_{T+1}^{\mathcal{M}^{\prime}}=0\right)$. We then have the following result:

Lemma 1 Consider a problem instance of the MSP as described above. Then a selection of markets has strictly positive profit if and only if it satisfies the even subsequence property.

Proof Note that all possible solutions for this market selection problem instance fall into one of the following four categories:

- select no markets, i.e., $\mathcal{M}^{\prime}=\varnothing$ :

Clearly, $\Pi\left(\mathcal{M}^{\prime}\right)=0$.

- select all markets, i.e., $\mathcal{M}^{\prime}=\mathcal{M}$ :

In this case the profit equals

$$
\Pi\left(\mathcal{M}^{\prime}\right)=\sum_{m=1}^{M} R_{m}-C(T)=\sum_{m=1}^{M} r \sum_{t=1}^{T} d_{t}^{m}-C(T)=r T-C(T)=0 .
$$

- $\mathcal{M}^{\prime} \notin\{\varnothing, \mathcal{M}\}$ and $\mathcal{M}^{\prime}$ does not satisfy the even subsequence property:

Let $k$ be the number of periods in the selection with unit demand, i.e., $k=$ $\sum_{t=1}^{T} \sum_{m \in \mathcal{M}^{\prime}} d_{t}^{m}$. As there is at least one odd subsequence of unit demands, 
it follows from (2) that the total cost $C\left(\mathcal{M}^{\prime}\right)$ equals at least $\frac{3}{2} k+\frac{1}{2}$. Therefore,

$$
\Pi\left(\mathcal{M}^{\prime}\right)=\sum_{m \in \mathcal{M}^{\prime}} R_{m}-C\left(\mathcal{M}^{\prime}\right) \leq r k-\left(\frac{3}{2} k+\frac{1}{2}\right)<r T-\left(\frac{3}{2} T+\frac{1}{2}\right)=0
$$

- $\mathcal{M}^{\prime} \notin\{\varnothing, \mathcal{M}\}$ and $\mathcal{M}^{\prime}$ satisfies the even subsequence property:

Again, let $k$ be the number of periods in the selection with unit demand. As we have only even subsequences of unit demands, the optimal lot-sizing solution has only subplans of 2 periods. This means that the average cost per unit demand equals $\frac{3}{2}$, and the optimal profit equals:

$$
\Pi\left(\mathcal{M}^{\prime}\right)=\sum_{m \in \mathcal{M}^{\prime}} R_{m}-C\left(\mathcal{M}^{\prime}\right)=r k-\frac{3}{2} k=k\left(r-\frac{3}{2}\right)>0 .
$$

This proves the desired result.

\subsection{Reduction from 3SAT}

Consider the following decision version of the market selection problem:

Market Selection Decision (MSD) problem: Is there a non-empty set of markets with profit strictly larger than $B$ ?

Note that Lemma 1 implies that when $B=0$, for problem instances of the MSP as described in Sect. 3.1, solving the MSD problem is equivalent to determining whether a selection of markets exists that satisfies the even subsequence property. Using a reduction from the $3 \mathrm{SAT}$ problem to this decision problem, we next show the following result:

Theorem 1 MSD is strongly $\mathcal{N} \mathcal{P}$-complete.

Proof It is easy to see that MSD $\in \mathcal{N} \mathcal{P}$ since a nondeterministic algorithm needs only guess a selection of markets and check in polynomial time whether that selection has a profit larger than $B$. In the following reduction we set $B=0$ and construct a problem instance as described in Sect. 3.1. To show that MSD is $\mathcal{N} \mathcal{P}$-complete it is sufficient to prove that MSD is $\mathcal{N} \mathcal{P}$-complete for this class of instances. Furthermore, it follows from Lemma 1 that MSD is $\mathcal{N} \mathcal{P}$-complete for this class of instances if the corresponding problem of finding a market selection that satisfies the even subsequence property is $\mathcal{N} \mathcal{P}$-complete.

The classical 3SAT problem is described as follows: Given a collection $C=$ $\left\{c_{1}, c_{2}, \ldots, c_{m}\right\}$ of clauses on a finite set $U$ of variables such that $\left|c_{i}\right|=3$ for $1 \leq i \leq m$, is there a truth assignment for $U$ that satisfies all the clauses in $C$ ? This problem is known to be $\mathcal{N} \mathcal{P}$-complete [12].

Before we proceed with the reduction, we make the following observations for any instance of the 3SAT problem. First, note that if $u$ is a variable in $U$, then $u$ and $\bar{u}$ are literals over $U$, and we will denote a generic literal by $l$. Then each clause $c_{i}$ can be represented as a disjunction of 2 subclauses $\alpha_{i}$ and $\beta_{i}$, where $\left|\alpha_{i}\right|=2$ and $\beta_{i}$ is a 
literal. Without loss of generality we let $\alpha_{i}=l_{1}^{i} \vee l_{2}^{i}$ and $\beta_{i}=l_{3}^{i}$ for each clause of the form $c_{i}=l_{1}^{i} \vee l_{2}^{i} \vee l_{3}^{i}$. Therefore, a truth assignment should satisfy

$$
\begin{aligned}
u_{j} \oplus \bar{u}_{j}=1, & j & =1,2, \ldots,|U| \\
l_{1}^{i} \vee l_{2}^{i}=\alpha_{i}, & i & =1,2, \ldots,|C| \\
\alpha_{i} \vee l_{3}^{i}=1, & i & =1,2, \ldots,|C|
\end{aligned}
$$

(where the $\vee$ operator corresponds to the logical "OR" operator, while the $\oplus$ operator corresponds to the logical "Exclusive OR" operator).

Now suppose we are given an instance of the 3SAT problem. We will construct an instance of the MSD problem that uses the cost and revenue parameters provided in Sect.3.1. The construction of this instance of the MSD will create a market for each literal and subclause in the 3SAT problem, where the selection of a market corresponds to a truth assignment for the corresponding literal or subclause. In addition, we will also create two "dummy markets" that are needed to ensure equivalence with 3SAT. More formally, we create the following markets:

- Create 2 markets for each variable in $U$ and its negation, i.e., $m_{u_{j}}$ and $m_{\bar{u}_{j}}(j=$ $1, \ldots,|U|)$.

- Create 1 market corresponding to a subclause $\alpha$ of each clause in $C$, i.e., $m_{\alpha_{i}}$ $(i=1, \ldots,|C|)$.

- Create 2 markets $m_{s}$ and $m_{n}$. (We will later show that $m_{s}$ must be selected and $m_{n}$ must not be selected in a market selection with the even subsequence property.)

Given these markets, we construct a specially structured demand matrix such that a truth assignment exists for the instance of 3SAT if and only if the MSD problem we construct contains a profitable selection of markets (i.e., satisfying the even subsequence property). Note that, since in each period there is exactly one market with positive (unit) demand, we can characterize all demands through a vector of market indices that indicates the market that has positive demand in the corresponding period.

- For the first 3 periods we introduce unit demands for markets

$$
\left[m_{n} m_{s} m_{s}\right]
$$

i.e., $d_{1}^{m_{n}}=1, d_{2}^{m_{s}}=1, d_{3}^{m_{s}}=1$ (and the remaining demands in periods 1,2 , and 3 are zero).

- For each variable $u_{j}, j=1,2 \ldots,|U|$, add 4 periods with a unit demand for markets

$$
\left[m_{u_{j}} m_{s} m_{\bar{u}_{j}} m_{n}\right]
$$

- For each clause $c_{i}, i=1,2 \ldots,|C|$, add 8 periods with a unit demand for markets

$$
\left[m_{l_{1}^{i}} m_{\alpha_{i}} m_{l_{2}^{i}} m_{l_{1}^{i}} m_{\alpha_{i}} m_{l_{2}^{i}} m_{n} m_{n}\right]
$$


and 8 additional periods with a unit demand for markets

$$
\left[m_{\alpha_{i}} m_{s} m_{l_{3}^{i}} m_{\alpha_{i}} m_{s} m_{l_{3}^{i}} m_{n} m_{n}\right]
$$

We next need to show that, given the instance of MSD we have defined, a selection of markets satisfying the even subsequence property exists if and only if a truth assignment exists for the corresponding 3SAT problem. With this in mind, we call a market selection feasible if and only if it satisfies the even subsequence property. Initially, we assume that $m_{s}$ is selected and $m_{n}$ is not selected for any feasible solution, and later verify that this must indeed hold. Under this assumption, observe that the demand pattern in (7) satisfies a feasible market selection if and only if the exclusive disjunction requirement in (3) is satisfied. The pattern in (8) then ensures that $m_{\alpha_{i}}$ is selected if and only if $m_{l_{1}^{i}}$ or $m_{l_{2}^{i}}$ or both are selected, which corresponds to the requirement in (4). Similarly, the pattern in (9) guarantees a feasible market selection if and only if (5) is satisfied by either selecting $m_{\alpha_{i}}$ or $m_{l_{3}^{i}}$ or both. Therefore, we can conclude that, assuming $m_{s}$ must be selected and $m_{n}$ must not be selected for any feasible solution, the MSD problem instance has a feasible market selection if and only if the 3SAT instance has a "true" assignment.

It remains to show that $m_{s}$ must be selected and $m_{n}$ not selected for any feasible solution.

- Assume that $m_{n}$ is selected. From (6), $m_{n}$ appears in the first period followed by a demand from $m_{s}$, and therefore $m_{s}$ must be selected as well. Selection of $m_{n}$ and $m_{s}$ together forces all introduced markets to be selected as a result of (7), (8) and (9). However, this solution is infeasible because the total number of periods is not even. (Selection of $m_{n}$ and $m_{s}$ together, along with the first sequence of the form of (7) imply that the only way to achieve an even subsequence among the first seven periods is if we select both $m_{u_{1}}$ and $m_{\bar{u}_{1}}$. Similar reasoning implies that each market $m_{u_{j}}$ and $m_{\bar{u}_{j}}$ must be selected. Finally, each market $m_{\alpha_{i}}$ must be selected as well to avoid a subsequence of size 3 (see columns 21-23 in Table 1) because of the market demands defined in (8) and (9).)

- Assume that neither $m_{s}$ nor $m_{n}$ is selected. Then (7), (8) and (9) require that none of the markets are selected and the result is an empty set, which is also infeasible. (If neither $m_{n}$ nor $m_{s}$ is selected, then we cannot select any $m_{u_{j}}$ or $m_{\bar{u}_{j}}$, or by (7) we would have a sequence of length 1 . Finally, given that no market is selected so far, we cannot select any $m_{\alpha_{i}}$ either, because again we would have a sequence of length 1 by (8) and (9).)

In conclusion, if 3SAT has a "true" assignment, then only $m_{s}$ must be selected among these two markets and the MSD problem results with a feasible market selection. The presented reduction is polynomial and the largest number involved is $\mathcal{O}(T)$. Hence, the decision version of the market selection problem is strongly $\mathcal{N} \mathcal{P}$-complete.

An example of the demand matrix resulting from the above reduction for a single clause is given in Table 1. Note that the cost parameters are time-invariant in the proof, 
Table 1 Demand matrix resulting from the clause $u_{1} \vee u_{2} \vee u_{3}=\alpha_{1} \vee u_{3}$

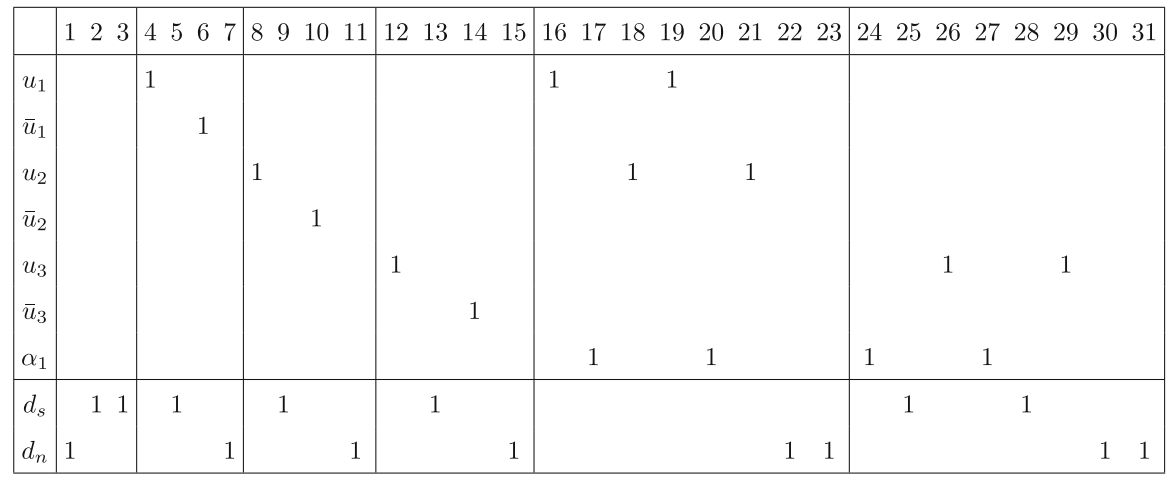

Every block indicated by the vertical lines corresponds to a sequence of market demands defined in (6), (7), (8) or (9)

i.e., we are considering the least general case for the cost parameters in terms of timedependency. Furthermore, the market revenue per demand unit $r_{m}$ is also constant for all markets (i.e., $r_{m}=r=R_{m} / \sum_{t=1}^{T} d_{t}^{m}$ ).

The proof of Theorem 1 is based on instances of the MSD in which the demand matrix is very sparse. However, by making a slight modification to the proof, we can show that the MSD is also $\mathcal{N} \mathcal{P}$-complete if we restrict ourselves to problem instances in which each market has positive demand in each period. In particular, for the class of problem instances of Sect.3.1, replace the zero demands by a positive value $\delta>0$. Furthermore, we add two time periods at the start of the planning horizon with unit demands for market $m_{s}$ and $\delta$ demands for the other markets. This is necessary because we do not want to setup in period 1 to satisfy a demand of $\delta$, as this causes a negative profit for any market selection. Finally, the revenue of market $m$ becomes $R_{m}=r \sum_{t=1}^{T}\left\lfloor d_{t}^{m}\right\rfloor$ with $r=3 / 2+1 /(2 T)$ (so $\delta$ demands do not contribute to the summation).

It can be verified that a market selection has a strictly positive profit if and only if the following properties are satisfied: (i) the selection has at least a unit demand in period 1 , (ii) the selection satisfies a modified even subsequence property where all subsequences of consecutive periods with demand at least 1 are of even length, and (iii) $\delta$ is sufficiently small (for example, $\delta=(r-3 / 2) /\left(M T^{2}\right)$ ). With the above modification, the problem instance preserves the property that any feasible truth assignment for the 3SAT instance corresponds to a market selection that satisfies the modified even subsequence property (and vice versa). Furthermore, because only market selections with properties (i)-(iii) have a strictly positive profit and $m_{s}$ has a unit demand in period 1 , the class of problem instances with only strictly positive demands is $\mathcal{N P}$-complete.

\subsection{Approximation results}

As we will show next, the proof of Theorem 1 in fact implies that it is unlikely that the solution to the MSP can be approximated efficiently. An algorithm is called a 
$(1-\varepsilon)$-approximation algorithm if, for any problem instance, the algorithm finds a solution with profit $\Pi_{A}$ that satisfies

$$
\Pi_{A} \geq(1-\varepsilon) \Pi^{*}
$$

where $\Pi^{*} \geq 0$ is the optimal profit and $\varepsilon>0$.

Theorem 2 There exists no polynomial-time $(1-\varepsilon)$-approximation algorithm for the $M S P$ for any $0<\varepsilon<1$ unless $\mathcal{P}=\mathcal{N} \mathcal{P}$.

Proof Consider a problem instance for the MSP reduced from a 3SAT instance as described in the proof of Theorem 1. Recall that any solution for the MSP instance with profit $\Pi>0$ corresponds to a feasible truth assignment for the 3SAT instance and that a solution with $\Pi \leq 0$ corresponds to an infeasible truth assignment. Assume there exists a polynomial-time $(1-\varepsilon)$-approximation algorithm $A$ for some $0<\varepsilon<1$. If $\Pi^{*}=0$, then the 3SAT instance is unsatisfiable and the profit found by algorithm $A$ satisfies $\Pi_{A} \geq(1-\varepsilon) \Pi^{*}=0$ and hence $\Pi_{A}=0$. If $\Pi^{*}>0$, then the 3SAT instance is satisfiable and the profit of the solution found by algorithm $A$ satisfies $\Pi_{A} \geq(1-\varepsilon) \Pi^{*}>0$. But this means that algorithm $A$ determines in polynomial time whether the 3SAT instance is satisfiable, which is a contradiction unless $\mathcal{P}=\mathcal{N} \mathcal{P}$.

Theorem 2 shows that the problem is inapproximable for any $0<\varepsilon<1$. Note that the algorithm that selects the empty set of markets has profit $\Pi=0$ and hence is a 0 -approximation. The inapproximability result is in contrast with the result of [22]. They consider a number of inventory and facility location models with market selection which includes the MSP. However, instead of maximizing profit, they minimize the following cost function:

$$
\sum_{m=1}^{M} R_{m}\left(1-z_{m}\right)+\sum_{t=1}^{T}\left(K_{t} y_{t}+\sum_{i=1}^{t} \sum_{m=1}^{M} c_{i, t}^{m} x_{i, t}^{m}\right) .
$$

In this objective function the lost revenue of a non-selected market is considered an opportunity cost. Note that from an optimization point of view, minimizing (10) is equivalent to maximizing (1a). Levi et al. [22] develop a polynomial-time 1.582approximation algorithm for the MSP.

At first sight, this approximation result seems inconsistent with the inapproximability result of Theorem 2 . However, this is not the case. Let $\Gamma\left(\mathcal{M}^{\prime}\right)$ be the cost for a market selection $\mathcal{M}^{\prime} \subset \mathcal{M}$ using (10) and let $\Pi\left(\mathcal{M}^{\prime}\right)$ be the profit for this market selection using (1a). Then the relationship

$$
\Gamma\left(\mathcal{M}^{\prime}\right)+\Pi\left(\mathcal{M}^{\prime}\right)=\sum_{m=1}^{M} R_{m}
$$

holds. Assume there exists a $(1+\varepsilon)$-approximation for the minimization problem for some $\varepsilon>0$. For a given MSP instance let $\mathcal{M}^{A}$ be the market selection found by this algorithm and let $\mathcal{M}^{*}$ be the optimal market selection. Then 


$$
\frac{\Gamma\left(\mathcal{M}^{A}\right)}{\Gamma\left(\mathcal{M}^{*}\right)} \leq 1+\varepsilon \Leftrightarrow \frac{\Pi\left(\mathcal{M}^{A}\right)}{\Pi\left(\mathcal{M}^{*}\right)} \geq(1+\varepsilon)-\varepsilon \frac{\sum_{m=1}^{M} R_{m}}{\Pi\left(\mathcal{M}^{*}\right)}
$$

As $\sum_{m=1}^{M} R_{m} / \Pi\left(\mathcal{M}^{*}\right)$ can be arbitrarily large, the $(1+\varepsilon)$-approximation algorithm for the minimum cost problem does not provide any performance guarantee for the maximum profit problem.

\subsection{Implication for economic lot-sizing games}

In this section we show the implication of the complexity result for economic lot-sizing (ELS) games (see [28] for the details). In the ELS game we have a set of players $\mathcal{M}$, where every player $m \in \mathcal{M}$ faces a lot-sizing problem. That is, every player $m$ has a demand $d_{t}^{m}$ in period $t=1, \ldots, T$ and it is assumed that setup costs $K_{t}$, production costs $p_{t}$ and holding costs $h_{t}$ are player independent. Note that we reuse some notation of the previous section to show the relation between the MSP and ELS games. If a coalition $\mathcal{M}^{\prime} \subset \mathcal{M}$ cooperates, then the cost of this coalition $C\left(\mathcal{M}^{\prime}\right)$ is equal to the optimal cost of the lot-sizing problem with demands $d_{t}^{\mathcal{M}^{\prime}}=\sum_{m \in \mathcal{M}^{\prime}} d_{t}^{m}$ and cost parameters $K_{t}, p_{t}$ and $h_{t}(t=1, \ldots, T)$. Because of economies of scale, all players have an incentive to cooperate in a single coalition $\mathcal{M}$, the so-called grand coalition.

The main question in a cooperative game is whether there exists a fair cost allocation among the players. The core of a game is often used as a solution concept, i.e., an allocation of the costs to the players, in cooperative game theory.

The core of the ELS game is defined as

$$
\left\{R \in \mathbb{R}^{M}: \sum_{m \in \mathcal{M}} R_{m}=C(\mathcal{M}), \sum_{m \in \mathcal{M}^{\prime}} R_{m} \leq C\left(\mathcal{M}^{\prime}\right) \text { for all } \mathcal{M}^{\prime} \subset \mathcal{M}\right\}
$$

where we let $C(\varnothing)=0$. Note that no coalition $\mathcal{M}^{\prime}$ has an incentive to leave the grand coalition for a cost allocation $R$ that is in the core. In the next paragraph it will be clear why we use the notation $R$ to represent the cost allocation, while it was used to represent revenues in Sect. 2. Several interesting questions are: (i) is the core of the game non-empty?, (ii) can a core element be found in polynomial time?, and (iii) can we check in polynomial time whether a cost allocation is in the core? [28] show that the core of an ELS game is non-empty. Using a very different approach, [25] answered (i) and (ii) affirmatively for a class of games defined on location problems. Since the ELS problem can be viewed as a specially structured facility location problem (see [19]), these results apply to the ELS games as well.

It is still an open question (see [6]) whether core membership of a cost allocation can be determined in polynomial time for the ELS games. We will provide a negative answer to this question. To this end, define a problem instance of the ELS game with the demand and cost parameters as in the problem instance of Sect. 3.1. Furthermore, consider the cost allocation $R$ with $R_{m}(m \in \mathcal{M})$ equal to the revenue of market $m$ in the problem instance of Sect. 3.1. Note that $R$ is a cost allocation of the ELS game because $\sum_{m \in \mathcal{M}} R_{m}=C(\mathcal{M})$. In fact, every instance of the MSP problem with the 
property $\sum_{m \in \mathcal{M}} R_{m}=C(\mathcal{M})$ corresponds to an instance of the ELS game with an associated cost allocation $R$ and vice versa. Clearly, the allocation $R$ is not in the core if there exists a coalition $\mathcal{M}^{\prime}$ such that $\sum_{m \in \mathcal{M}^{\prime}} R_{m}>C\left(\mathcal{M}^{\prime}\right)$. Finding such a coalition is one of the subproblems in [8], who develop a row generation procedure to determine a core element of the ELS game. Using the relation to the MSP instances of Sect. 3.1, it is clear that finding such a coalition is equivalent to finding a market selection with a strictly positive profit, which is strongly $\mathcal{N} \mathcal{P}$-complete by Theorem 1. This leads to the following corollary:

Corollary 1 It is strongly $\mathcal{N} \mathcal{P}$-complete to determine whether a cost allocation of the ELS game is in the core of the game.

\section{Polynomially solvable cases}

Although the general MSP is $\mathcal{N} \mathcal{P}$-hard, in this section we will present some practical special cases for which the MSP can be solved in polynomial time. We will use different techniques and results from the literature to solve these cases. For example, one case can be solved by finding a maximum-flow/minimum-cut in an appropriately defined network, while other cases will be solved through dynamic programming (DP).

\subsection{Seasonal demand}

Consider a set of markets that only differ in demand volume but that experience the same seasonal demand fluctuations, i.e., $d_{t}^{m}=\sigma_{t} d_{m}$ where $\sigma_{t}$ is the seasonal coefficient for period $t$ and $d_{m}$ represents the demand volume of market $m$. We will call this the seasonal demand case and we will show that this case can be solved in polynomial time.

To this end, consider the following parametric lot-sizing problem. First, let the base demand vector be given by $\sigma=\left(\sigma_{1}, \ldots, \sigma_{T}\right)$. Then let $C_{S}(d)$ be the cost of a lotsizing problem with demand vector $\sigma d$. For a given production plan (i.e., fixing the production periods), the lot-sizing cost increases linearly in $d$, since it is exactly know from which period a demand is satisfied because of the zero-inventory property.

Because $C_{S}(d)$ is the lower envelope of the cost functions of all production plans, it is piecewise linear and concave.

This means that for the seasonal case we can rewrite the MSP as

$$
\begin{aligned}
&\left(\mathrm{P}_{S}\right) \max \sum_{m=1}^{M} R_{m} z_{m}-C_{S}\left(\sum_{m=1}^{M} d_{m} z_{m}\right) \\
& \text { s.t. } \quad z_{m} \in\{0,1\}, \quad m=1, \ldots, M .
\end{aligned}
$$

Huang et al. [18] show that for any concave function $C_{S}$, the optimal solution to $\left(\mathrm{P}_{S}\right)$ can be found in polynomial time. In particular, they show that if we re-order the markets in nonincreasing order of the ratio $R_{m} / d_{m}$, an optimal solution to $\left(\mathrm{P}_{S}\right)$ can be found among the market selections $\{1, \ldots, m\}$ for $m=0, \ldots, M$. Since the ordering of the 
markets takes $\mathcal{O}(M \log M)$ time and because we have to solve $M$ lot-sizing problems, the total running time to solve the seasonal demand case is $\mathcal{O}(M(\log M+T \log T))$.

\subsection{Market-specific prices}

We next consider instances of the MSP where the demand for market $m$ in period $t$ is given by

$$
d_{t}^{m}=\alpha_{t}-\beta_{t} w_{m}
$$

where $\alpha_{t}$ and $\beta_{t}$ are time-dependent coefficients of a linear price-demand response curve and $w_{m}$ is the price in market $m$. Note that we consider each of the market prices to be fixed, i.e., they are not themselves decision variables in our problem. Note also that, more generally, $w_{m}$ could represent any market-dependent but stationary function of price, allowing for more general price-demand response curves than the linear one presented above.

The aggregate demand in period $t$ corresponding to a given market selection $z$ is given by

$$
\sum_{m=1}^{M}\left(\alpha_{t}-\beta_{t} w_{m}\right) z_{m}=\alpha_{t} \sum_{m=1}^{M} z_{m}-\beta_{t} \sum_{m=1}^{M} w_{m} z_{m}
$$

Once again we consider a parametric lot-sizing problem; in this case one where the demand in period $t$ is given by $\alpha_{t} k-\beta_{t} p$, and where the parameters are $k$ and $p$. Let $C_{P}(k, p)$ be the corresponding lot-sizing cost function. The MSP can then be written as

$$
\begin{aligned}
\left(\mathrm{P}_{P}\right) & \max \\
\text { s.t. } & \sum_{m=1}^{M} R_{m} z_{m}-C_{P}\left(\sum_{m=1}^{M} z_{m}, \sum_{m=1}^{M} w_{m} z_{m}\right) \\
& \left.z_{m} \in, 1\right\}, \quad m=1, \ldots, M .
\end{aligned}
$$

Now suppose that we know that, in the optimal solution to $\left(\mathrm{P}_{P}\right)$, exactly $\bar{k}$ markets are selected. The problem then reduces to

$$
\begin{aligned}
\left(\mathrm{P}_{P}(\bar{k})\right) \max & \sum_{m=1}^{M} R_{m} z_{m}-C_{P}\left(\bar{k}, \sum_{m=1}^{M} w_{m} z_{m}\right) \\
\text { s.t. } & \sum_{m=1}^{M} z_{m}=\bar{k}, \\
& z_{m} \in\{0,1\}, \quad m=1, \ldots, M .
\end{aligned}
$$

As in Sect. 4.1, it is easy to see that $C_{P}(\bar{k}, \cdot)$ is a concave function. The algorithm of [23] now solves this problem in $\mathcal{O}\left(M^{2}(\log M+T \log T)\right)$ time (where the term 
$T \log T$ represents the time required to solve a lot-sizing problem). This result immediately implies that, by solving $\left(\mathrm{P}_{P}(\bar{k})\right)$ for $\bar{k}=1, \ldots, M$, we can find the optimal solution to $\left(\mathrm{P}_{P}\right)$ in $\mathcal{O}\left(M^{3}(\log M+T \log T)\right)$ time.

However, we can reduce the running time of this algorithm by a factor of $M$ by employing the similarities between the $M$ problems of the form $\left(\mathrm{P}_{P}(\bar{k})\right)$ that need to be solved. This yields a generalization of the approach of [23] to solve $\left(\mathrm{P}_{P}\right)$. This result is summarized in the following theorem:

Theorem 3 The MSP with market-specific prices can be solved in $\mathcal{O}\left(M^{2}(\log M+\right.$ $T \log T))$ time.

Proof See the Appendix.

\subsection{Infinite holding cost}

Assume that the manufacturer does not want to or cannot hold inventory, for example because holding costs are high or there is no storage space available. We will call this case the infinite holding cost case. Since no inventory is carried forward, we only need the decision variable $x_{i, t}^{m}$ with $i=t$ in the formulation of Sect. 2, which reduces the number of variables significantly. For a given selection of markets, the setups will occur in the periods where at least one of the selected markets has a strictly positive demand. Furthermore, for each market, the variable production cost can be subtracted from the revenue because any demand is satisfied from the period in which it occurs and hence the variable cost is known. This means that a problem instance only consists of demand, revenue and setup cost parameters. The profit $\Pi\left(\mathcal{M}^{\prime}\right)$ for some subset of markets $\mathcal{M}^{\prime} \subset \mathcal{M}$ equals

$$
\Pi\left(\mathcal{M}^{\prime}\right)=\sum_{m \in \mathcal{M}^{\prime}} R_{m}-\sum_{\left\{t: d_{t}^{m}>0 \forall m \in \mathcal{M}^{\prime}\right\}} K_{t} .
$$

It turns out that the class of infinite holding cost problems is equivalent to a class of selection problems in the literature (see [17] for a survey on this class of problems). This class of selection problems can be described as follows. We are given a set of items $\mathcal{I}$ and a collection of subsets $S_{j} \subset \mathcal{I}$ with $j \in \mathcal{J}$. There is a cost $c_{i}$ associated with each item $i \in \mathcal{I}$ and a benefit $b_{j}$ associated with each subset $S_{j}(j \in \mathcal{J})$. The items corresponding to some subcollection $\mathcal{J}^{\prime} \subset \mathcal{J}$ are $\left\{i \in S_{j}: j \in \mathcal{J}^{\prime}\right\}$. The profit of subcollection $\mathcal{J}^{\prime}$ equals the benefits of the subsets minus the cost of the items in the subsets, i.e.,

$$
\sum_{j \in \mathcal{J}^{\prime}} b_{j}-\sum_{\left\{i \in S_{j}: j \in \mathcal{J}^{\prime}\right\}} c_{i}
$$

The objective is to find a subcollection $\mathcal{J}^{\prime}$ that maximizes the profit.

We will show that an infinite holding cost instance of the MSP corresponds to an instance from the class of selection problems. First, the time periods form the set $\mathcal{I}$ 
and the markets form the set $\mathcal{J}$. Second, the $\cos t c_{i}$ is set to the setup cost of the period corresponding to $i$ and the benefit $b_{j}$ is set to the revenue of the market corresponding to $j$. Finally, the set $S_{j}$ consists of the periods for which the market corresponding to $j$ has strictly positive demands. So every infinite holding cost instance can be transformed to an instance from the class of selection problems. It is not difficult to see that the reverse also holds.

The class of selection problems can be solved in polynomial time by solving a max-flow/min-cut problem on an appropriately defined network. When applied to the infinite holding cost case, the network consists of $\mathcal{O}(M+T)$ nodes and $\mathcal{O}(M T)$ arcs. As the max-flow/min-cut problem can be solved in polynomial time, the infinite horizon MSP can be solved in polynomial time. For more details on the class of selection problems we refer to [17] and its references.

\subsection{Selection of orders spanning up to $k$ consecutive periods}

In this section we begin by considering the special case in which each market has demand in only one period of the planning horizon; thus, the selection of a market is equivalent to selecting one customer order in a single period. This means that the problem decomposes by time period and allows for a polynomial-time solution via dynamic programming, as shown in [13]. They consider an equivalent pricing and lot-sizing model where, in each period, demand depends on the price level. The goal is to find a sequence of prices and a production plan that maximize total profit. They assume that the revenue function in each period is piecewise-linear and concave in the demand level, and show that the problem can be interpreted as an equivalent order selection problem. Geunes et al. [13] solve this problem by using the fact that an optimal solution consists of a series of subplans. For a given subplan, only the orders for which the revenue per demand unit exceeds the variable cost per demand unit are satisfied. The total running time of this approach is $\mathcal{O}\left(M T^{2}\right)$. This of course immediately implies that that the MSP with exactly one positive demand period for each market can be solved in $\mathcal{O}\left(M T^{2}\right)$ time.

An interesting generalization of this case allows for a customer's order to span at most $k$ consecutive periods for some fixed $1<k<T$. That is, in this case each "order" corresponds to a market with zero demands except possibly within a sequence of $k$ consecutive periods. Formally, for every market $m \in \mathcal{M}$ there exists a time period $t_{m}$ with $d_{i}^{m}=0$ for $i=1, \ldots, t_{m}-1$ and $i=t_{m}+k, \ldots, T$. We next show that this problem can, for constant $k$, also be solved in polynomial time using DP.

To this end, let $\mathcal{M}_{t}(t=k, \ldots, T)$ be the set of markets whose first positive demand period is period $t-k+1$ (we use the convention that $\mathcal{M}_{T}$ denotes the set of markets whose first positive demand is in one of the periods $T-k+1, \ldots, T)$. We know that demands for markets in the set $\mathcal{M}_{t}$ can only occur within the periods $\{t-k+1, \ldots, t\}$ for $t \geq k$. Furthermore, let $\mathcal{P}_{t}$ be the set of partial production plans that span periods $i, \ldots, t$ for some $i=1, \ldots, t$, where $i$ is the last setup period in periods $\{1, \ldots, t-k+1\}$ and the remaining setups in the partial plan are in $\{t-k+2, \ldots, t\}$. Note that we are only considering partial plans here, so that there may be more setups in periods $\{1, \ldots, t-k+1\}$ in a final solution that contains a partial plan from $\mathcal{P}_{t}$. 
Furthermore, observe that if market $m \in \mathcal{M}_{t}$ is selected in an optimal solution, it must be satisfied using the setups from some partial plan $P \in \mathcal{P}_{t}$ (observe that if a market in $\mathcal{M}_{t}$ has demand in period $t-k+1$ and we use a partial plan $P \in \mathcal{P}_{t}$, then the only available setup to satisfy demand in period $t-k+1$ is the first setup in the partial plan $P \in \mathcal{P}_{t}$, which is the last setup among periods $\{1, \ldots, t-k+1\}$; all other demands for the market must be satisfied using this setup or using available setups in the partial plan from periods $\{t-k+2, \ldots, t\})$. We let $P_{\tau}=1$ if $\tau$ is a production period in partial plan $P$ and $P_{\tau}=0$ otherwise; thus $P \in \mathcal{P}_{t}$ is a binary vector of length $t-i+1$ with elements $P_{\tau}, \tau=i, \ldots, t$ (note that vectors in $\mathcal{P}_{t}$ may have different lengths). Finally, we let $t_{P}^{1}=i$ be the first production period of $P$ (and by definition the only production period of $P$ in $\{1, \ldots, t-k+1\})$.

We will first focus on the cost associated with a given partial plan. We let $c(m, P)$ be the variable production and holding cost incurred when satisfying the demands of market $m \in \mathcal{M}_{t}$ using the setups in partial plan $P \in \mathcal{P}_{t}$. Because of the zero-inventory property, these costs are easy to calculate. Suppose that the partial plan $P$ is part of the optimal solution. Then market $m$ will be selected only if $R_{m} \geq c(m, P)$. So if we let $\Pi(t, P)$ be the profit (excluding setup cost) when considering all markets in $\mathcal{M}_{t}$ using partial plan $P \in \mathcal{P}_{t}$, then

$$
\Pi(t, P)=\sum_{m \in \mathcal{M}_{t}}\left(R_{m}-c(m, P)\right)^{+} .
$$

The forward DP we will present considers each solution as a sequence of "compatible" partial plans, and is based on the fact that if $P \in \mathcal{P}_{t}$ is part of the optimal solution, then either one of the following cases holds: (i) partial plan $P$ overlaps some partial plan $Q$, i.e., $Q \in \mathcal{P}_{j}$ with $t_{P}^{1} \leq j<t$, or (ii) there is a partial partial plan $Q$ that precedes $P$, i.e., $Q \in \mathcal{P}_{j}$ with $j<t_{P}^{1}$. Note that if $P$ overlaps some partial plan $Q \in \mathcal{P}_{j}$ with $j<t$, then $P$ also overlaps with some partial plan $Q \in \mathcal{P}_{t-1}$. Furthermore, partial plans $P \in \mathcal{P}_{t}$ and $Q \in \mathcal{P}_{t-1}$ need to be "compatible". That is, period $i$ is a production period in $P$ if and only if $i$ is a production period in $Q$. Formally, we have $Q_{i}=P_{i}$ for $i=t-k-2, \ldots, t-1$ and either $t_{P}^{1}=t_{Q}^{1}$ or $t_{P}^{1}=t-k+1$ and $Q_{t-k+1}=1$. We denote the compatibility of partial plans $P$ and $Q$ by $P \sim Q$.

We are now ready to state the recursion formulas. Let $F(t, P)$ be the optimal profit when considering markets $\bigcup_{i=k}^{t} \mathcal{M}_{i}$ and with partial plan $P \in \mathcal{P}_{t}$ contained in the solution. Furthermore, let $F(t)$ be the optimal profit up to period $t$. Then, for $t=k, \ldots, T$ and $P \in \mathcal{P}_{t}$ we have

$$
F(t, P)=\max \left\{\begin{array}{l}
\max _{\left\{Q \in \mathcal{P}_{t-1}: Q \sim P\right\}}\left\{F(t-1, Q)+\Pi(t, P)-P_{t} K_{t}\right\}, \\
F\left(t_{P}^{1}-1\right)+\Pi(t, P)-\sum_{i=t_{p}^{1}, \ldots, t} P_{i} K_{i}
\end{array}\right.
$$

and

$$
F(t)=\max \left\{F(t-1), \max _{P \in \mathcal{P}_{t}}\{F(t, P)\}\right\} .
$$

Note that (11) considers the two cases mentioned in the previous paragraph. To prevent double counting the setup costs in cases involving overlapping partial production plans, we only subtract the setup cost of the production period in period $t$ (if any). 
To initialize the DP algorithm, we let $\mathcal{P}_{k-1}=\varnothing$ and $F(t)=0$ for $t=0, \ldots, k-1$. Clearly, the optimal profit equals $F(T)$.

Finally, we determine the running time of the DP algorithm. First, note that $\left|\mathcal{P}_{t}\right|=$ $(t-k+1) 2^{k-1}=\mathcal{O}(T)$ for fixed $k$. It takes $\mathcal{O}(k)=\mathcal{O}(1)$ time to calculate the variable cost associated with market $m \in \mathcal{M}_{t}$ for some $P \in \mathcal{P}_{t}$, which means that all values $c(m, t, P)$ can be determined in $\mathcal{O}\left(M T^{2}\right)$ time. Furthermore, for a given $t$ and $P \in \mathcal{P}_{t}$, the calculation of (11) takes $\mathcal{O}(T)$ time as $\left|\mathcal{P}_{j}\right|=\mathcal{O}(T)$. So the calculation of all values $F(t, P)$ takes $\mathcal{O}\left(T^{3}\right)$ time. Finally, it takes $\mathcal{O}\left(T^{2}\right)$ time to determine all values $F(t)$ in (12). Therefore, the total running time is $\mathcal{O}\left(M T^{2}\right)+\mathcal{O}\left(T^{3}\right)+\mathcal{O}\left(T^{2}\right)=$ $\mathcal{O}\left(T^{2}(M+T)\right)$.

\subsection{Staircase demand matrix}

The final special case that we will consider deals with a situation in which, in each period, there is positive demand in only one market, and each market has positive demand in a consecutive sequence of periods. (Note that this is a special case of the class of instances for which the corresponding MSP was shown to be $\mathcal{N} \mathcal{P}$-complete.) This situation may occur, for example, if we face demands in a single market only, but the planning horizon is partitioned into a collection of time intervals with the property that if we satisfy demand in a period we have to satisfy demand in all periods that are in the same interval. These intervals could correspond to seasons, so that this constraint would, for example, say that we should either satisfy demand for the entire summer season or not satisfy demand in any of the summer months. Now if we view each time interval as a distinct "market", we obtain the more general situation sketched above. This class of problems has the attractive property that, in contrast with the general MSP, there is a natural ordering for the markets based on the demands.

More formally, let market $m$ have positive demand in periods $t_{1}^{m}, \ldots, t_{2}^{m}$. Furthermore, assume that $t_{2}^{m}<t_{1}^{m+1}$ for $m=1, \ldots, M-1$. This ensures that for two different markets the positive demand periods do not have any "overlap" and the positive values in the demand matrix form a "staircase." As in the previous sections we will solve this problem by DP. Define $\Pi(m, t)\left(m=1, \ldots, M, t=1, \ldots, t_{2}^{m}\right)$ as the maximum profit when markets $1, \ldots, m$ are considered, and where the last setup occurs in period $t$. To develop the recursion, we will need some more notation. Let $c_{q}^{V}(s, t)$ be the variable production and holding cost to satisfy demand in periods $\{s, \ldots, t\}$ from production in period $q$ and let $c^{L S}(s, t)$ the optimal lot-sizing cost for the problem consisting of periods $\{s, \ldots, t\}$.

Consider the following two cases to calculate $\Pi(m, t)$ :

- $1 \leq t<t_{1}^{m}$ :

In this case we only have to determine whether the variable costs are less than the revenue for the market as the setup cost is already taken into account. Therefore,

$$
\Pi(m, t)=\Pi(m-1, t)+\left(R_{m}-c_{t}^{V}\left(t_{1}^{m}, t_{2}^{m}\right)\right)^{+}
$$


- $t_{1}^{m} \leq t \leq t_{2}^{m}$ :

Let $s$ be the first setup in $\left\{t_{1}^{m}, \ldots, t_{2}^{m}\right\}$. Furthermore, let $q$ be the last setup period before this interval ( $q=0$ if it does not exist). With this information we can calculate all relevant costs: the variable cost to satisfy demand in periods $\left\{t_{1}^{m}, \ldots, s-1\right\}$, the cost of the lot-sizing problem in periods $\{s, \ldots, t-1\}$, and the variable cost to satisfy demand in periods $\left\{t, \ldots, t_{1}^{m}\right\}$. Therefore, the recursion becomes

$$
\begin{gathered}
\Pi(m, t)=\max _{0 \leq q<t_{1}^{m}, t_{1}^{m} \leq s \leq t}\left\{\Pi(m-1, q)+\left(R_{m}-\left(c_{q}^{V}\left(t_{1}^{m}, s-1\right)+c^{L S}(s, t-1)\right.\right.\right. \\
\left.\left.\left.+c_{t}^{V}\left(t, t_{2}^{m}\right)\right)\right)^{+}-K_{t}\right\} .
\end{gathered}
$$

The recursion is initialized by $\Pi(0, t)=-\infty(t=1, \ldots, T)$ and $\Pi(m, 0)=0$ $(m=0, \ldots, M)$. Furthermore, for the recursion to be valid, we let $c_{s}^{V}(t, t-1)=$ $c^{L S}(t, t-1)=0(t=1, \ldots, T)$ and $c_{0}^{V}(s, t)=\infty$ for $s \neq t_{1}^{m}(m=1, \ldots, M)$. The optimal profit equals

$$
\Pi^{*}=\max _{t=0, \ldots, T}\{\Pi(M, t)\}
$$

Note that we can calculate the values $c_{q}^{V}(s, t)(1 \leq q \leq s \leq t)$ and $c^{L S}(s, t)(1 \leq s \leq$ $t)$ in $\mathcal{O}\left(T^{3}\right)$ and $\mathcal{O}\left(T^{2} \log T\right)$, respectively. Given these values and given a fixed $m$ and $t$, the calculation of $\Pi(m, t)$ for $1 \leq t<t_{1}^{m}$ and for $t_{1}^{m} \leq t \leq t_{2}^{m}$ takes $\mathcal{O}(1)$ and $\mathcal{O}\left(T^{2}\right)$ time, respectively. Therefore, the total running time of the DP is $\mathcal{O}\left(M T^{2}\right)$.

\section{Heuristics}

Because the MSP is $\mathcal{N} \mathcal{P}$-complete, it is very unlikely that there exist efficient algorithms to solve the problem to optimality. Moreover, the inapproximability result of Theorem 2 shows that we cannot hope for a polynomial-time approximation algorithm. Therefore, finding good solutions for large instances in a reasonable amount of time requires restricting ourselves to heuristics. In the following sections we propose two heuristics.

\subsection{An iterative algorithm}

Recall from Sect. 2 that given a selection of markets, the MSP is easy to solve, and given a production plan, the MSP is also easy to solve. This suggests the following iterative algorithm (IA). Start with an initial production plan $y$. Given this production plan, find the optimal set of markets, say $z=z(y)$. Given this selection of markets, determine the optimal production plan, say $y=y(z)$. Repeat this procedure until it converges. The idea of iterating between two types of variables is based on [20] and [29], who developed an algorithm that iterates between prices and production plans for a joint lot-sizing and pricing model.

In each iteration of the IA the profit strictly improves until identical production plans are found in two consecutive iterations. As the total number of production plans 
and market selections are finite, the algorithm terminates after a finite number of iterations. Furthermore, the algorithm terminates in some local optimal optimum $(\hat{y}, \hat{z})$. If we let $\pi(y, z)$ be the profit of the solution $(y, z)$, then a locally optimal solution $(\hat{y}, \hat{z})$ satisfies the property that $\pi(\hat{y}, \hat{z}) \geq \pi(y, \hat{z})$ for all $y \in\{0,1\}^{T}$ and $\pi(\hat{y}, \hat{z}) \geq \pi(\hat{y}, z)$ for all $z \in\{0,1\}^{M}$. So clearly, if we happen to come across either the production plan or the set of markets that are in an optimal solution, we will have found a (global) optimal solution.

In a single iteration we have to find the optimal selection of markets given a production plan and an optimal production plan given a selection of markets. Given a production plan, a market $m$ is selected if the variable production and holding cost are less than the revenue. For a single market, this takes $\mathcal{O}(T)$ time and hence $\mathcal{O}(M T)$ in total. Given a selection of markets, we have to solve a lot-sizing problem, which can be done in $\mathcal{O}(T \log T)$ time. Therefore, each iteration takes $\mathcal{O}(T(M+\log T))$ time.

It is not clear in advance how many iterations the IA will take. Clearly, this depends on the starting production plan $y$ (among other factors). In the implementation we used $T$ initial production plans $y^{(n)}(n=1, \ldots, T)$ where plan $y^{(n)}$ has $n$ subplans (approximately) equally spaced over the periods. Formally, plan $y^{(n)}$ has setups in periods $t=1+\left\lfloor\frac{i-1}{n} T\right\rfloor$ for $i=1, \ldots, n$. Furthermore, for instances with zero demands, we include some additional starting production plans. If $t$ is the first non-zero demand period for some market $m$, then we add starting production plans with the first setup in $t$, and $T-t-i+1$ (for $i=0, \ldots, T-t$ ) setups evenly distributed over periods $t, \ldots, T$ as described before. These production plans may be good starting points because an optimal solution will not have a setup period before the first non-zero demand period (in the case of time-independent cost parameters). So for an instance with only non-zero demands we have $T$ starting points and for instances with zero demands we have $\mathcal{O}(M T)$ starting points. Because we use multiple starting points, we stand a good chance of finding an optimal solution for problems where only a few locally optimal solutions exist. (Note that $\mathcal{M}^{\prime}=\varnothing$ is always a local optimum.)

\subsection{A rounding procedure}

As mentioned in Sect. 3.3, [22] developed a polynomial-time approximation algorithm for the MSP with (10) as the objective function. The approximation algorithm works as follows. First, the LP relaxation of $(P)$ is solved (with (10) as the objective function). We then sort the markets in nondecreasing order of their $z$-value in the optimal solution of the LP relaxation, say $z_{m}^{L P}, m=1, \ldots, M$. In the last step the algorithm chooses the best among the market selections $\{1, \ldots, m\}$ for $m=0, \ldots, M$. Levi et al. [22] show that this is a 1.582-approximation algorithm in terms of the cost of the solution. Although, as we mentioned before, this procedure does not provide a worst-case performance guarantee for the maximization version of the MSP, we will nevertheless apply the rounding procedure and empirically test its performance. Since, for each of the $\mathcal{O}(M)$ candidate solutions, we effectively round the market selection variables to 0 or 1 according to some threshold, we will refer to this algorithm as the rounding procedure (RP). Note that in the RP we have to solve one LP, sort $M$ numbers, and solve at most $M$ lot-sizing problems, and hence the RP takes polynomial time. 


\section{Computational study}

In this section we present the results of a computational study that tests the performance of the heuristics in terms of solution quality and computation time. Since Theorem 1 shows that the MSP is hard even when the cost parameters are time-invariant, we restrict ourselves to such instances; in particular, we set $p_{t}=p=0$ and $h_{t}=h=1$ throughout. Furthermore, such instances are the most common/realistic ones from a practical point of view, since technologies and holding cost rates are not expected to change often over time. Moreover, we performed preliminary testing on a random sample of problems with time dependent parameters and these tests show that instances with time-invariant cost parameters also tend to be the hardest ones.

We tested the heuristics on three sets of problem instances with randomly generated demands according to a

A. stationary demand distribution;

B. demand distribution that allows for a high proportion of periods with zero demand;

C. demand distribution that follows a seasonal pattern.

In many practical applications, we would expect a substantial number of periods with positive demands (Sets A and C). However, we also test the heuristics' performance on problem instances with a substantial number of zero-demands (Set B). So these instances are 'closer' to the instances presented in the $\mathcal{N} \mathcal{P}$-completeness proof of Sect. 3. All computations were performed on a $3.4 \mathrm{GHz}$ Pentium IV desktop computer with $2.0 \mathrm{~Gb}$ RAM and the Windows XP operating system. Furthermore, we used CPLEX 10.1 with default settings to solve $(P)$ and its LP-relaxation. As we mentioned before, we can relax one of the sets of binary constraints (1e) and (1f). Based on preliminary tests, we concluded that CPLEX could solve $(P)$ most efficiently when constraints (1e) are relaxed for instances in which $T>M$ and when constraints (1f) are relaxed when $M \geq T$. Because the results for Set $\mathrm{C}$ are very similar to the results of Set A, we only present the results for Sets A and B.

\subsection{Problem set A: Stationary demands}

In Set $\mathrm{A}$, the demands are generated from an integer uniform distribution over the interval $[0,2 \bar{d}]$, which we denote by $\mathrm{U}[0,2 \bar{d}]$. To obtain comparable problem instances for different values of $M$ and $T$, we let the parameters $K_{t}$ and $R_{m}$ depend on $M$ and $T$. In particular, we set $K_{t}=K=\alpha M \bar{d}$, where $\alpha$ is a parameter that characterizes the class of problem instances. We next discuss the implications of this choice of $\alpha$ on the total cost in the case that all markets are selected. The average demand per period equals $M \bar{d}$. In an EOQ model environment the optimal solution has a setup in every $n=\sqrt{2 \alpha}$ periods. In turn, a subplan of $n$ periods in a discrete environment has inventory and setup costs equal to

$$
\frac{1}{2} n(n-1) M \bar{d}+\alpha M \bar{d} .
$$


Table 2 Set A: Performance of the IA and RP heuristic

\begin{tabular}{|c|c|c|c|c|c|c|c|c|c|c|c|c|c|}
\hline \multirow[t]{2}{*}{ M } & \multirow[t]{2}{*}{$\mathrm{T}$} & \multirow[t]{2}{*}{$\alpha$} & \multirow[t]{2}{*}{ NS } & \multirow[t]{2}{*}{ LP } & \multicolumn{2}{|l|}{ IG } & \multicolumn{2}{|c|}{ avg $\operatorname{dev}(\%)$} & \multicolumn{2}{|c|}{$\max \operatorname{dev}(\%)$} & \multicolumn{2}{|c|}{ non-opt } & \multirow[t]{2}{*}{ IA-RP } \\
\hline & & & & & avg & $\max$ & IA & RP & IA & $\mathrm{RP}$ & IA & $\mathrm{RP}$ & \\
\hline \multirow[t]{4}{*}{40} & \multirow[t]{4}{*}{40} & 2 & 17 & 2 & 5.99 & 85.2 & 0.01 & 17.3 & 0.14 & 100.0 & 1 & 16 & $16-0$ \\
\hline & & 5 & 20 & 2 & 0.44 & 2.7 & 0.00 & 5.4 & 0.00 & 82.9 & 0 & 11 & $11-0$ \\
\hline & & 8 & 19 & 1 & 0.23 & 1.1 & 0.00 & 1.1 & 0.00 & 11.2 & 0 & 9 & $9-0$ \\
\hline & & 11 & 24 & 0 & 0.52 & 3.5 & 0.00 & 0.6 & 0.00 & 5.3 & 0 & 10 & $10-0$ \\
\hline \multirow[t]{4}{*}{80} & \multirow[t]{4}{*}{40} & 2 & 19 & 3 & 0.74 & 3.6 & 0.00 & 26.8 & 0.00 & 100.0 & 0 & 19 & $19-0$ \\
\hline & & 5 & 24 & 0 & 1.16 & 12.9 & 0.00 & 11.9 & 0.00 & 100.0 & 0 & 24 & $24-0$ \\
\hline & & 8 & 19 & 2 & 0.69 & 4.3 & 0.00 & 0.5 & 0.00 & 4.7 & 0 & 12 & $12-0$ \\
\hline & & 11 & 21 & 0 & 0.37 & 2.4 & 0.00 & 1.0 & 0.00 & 6.1 & 0 & 15 & $15-0$ \\
\hline \multirow[t]{4}{*}{40} & \multirow[t]{4}{*}{80} & 2 & 14 & 5 & 0.19 & 1.1 & 0.00 & 9.2 & 0.00 & 75.3 & 0 & 13 & $13-0$ \\
\hline & & 5 & 19 & 2 & 10.06 & 212.2 & 0.00 & 7.7 & 0.00 & 100.0 & 0 & 16 & $16-0$ \\
\hline & & 8 & 18 & 4 & 0.32 & 1.9 & 0.00 & 0.1 & 0.03 & 1.7 & 1 & 4 & $3-0$ \\
\hline & & 11 & 18 & 4 & 0.40 & 2.4 & 0.04 & 0.6 & 0.97 & 10.3 & 1 & 8 & $7-0$ \\
\hline
\end{tabular}

As there are $\frac{T}{n}$ subplans and $\alpha=\frac{1}{2} n^{2}$, the total cost equals approximately $\frac{1}{2} M T \bar{d}(2 \mathrm{n}-1)$ if all markets are selected. Finally, revenues are generated from $\mathrm{U}[0,2 \bar{R}]$ with $\bar{R}=\frac{1}{2} T \bar{d}(2 n-1)$. By using these parameters the profit will be approximately equal to zero when all markets are selected (and exactly zero when no market is selected).

We generated 25 instances with $\alpha \in\{2,5,8,11\}$ and $(M, T) \in\{(40,40),(80,40)$, $(40,80)\}$. The most important statistics on the quality of the heuristic solutions can be found in Table 2. Column 'NS' presents the number of times the optimal solution consists of a "non-straightforward" market selection, i.e., neither the empty selection nor the selection that includes all markets. Column 'LP' shows how many times the LP relaxation is tight and hence provides an optimal solution. The columns under ' $\mathrm{IG}$ ' provide the average (avg) and maximum (max) integrality gap (which is defined as the difference between the optimal values of the LP relaxation and the IP as a fraction of the optimal value of the IP). The columns 'avg dev' and 'max dev' give the average and maximum relative deviation from the optimal profit (in \%). The columns under 'non-opt' present the number of instances for which each of the heuristics does not find an optimal solution. Finally, a cell with value $a-b$ in column 'IA-RP' means that there are $a$ instances for which the IA finds a strictly better solution than the RP and $b$ instances for which the RP finds a strictly better solution than the IA.

Table 2 shows that the instances vary in terms of optimal solution characteristics. Most of the instances have an optimal solution that is a "non-straightforward" market selection. However, the integrality gap varies widely: in some instances the LP relaxation is tight (and gives the optimal solution), while in other instances the integrality gap is very large. Overall, Table 2 shows that the IA performs extremely well on problem instances in Set A. Only in 3 out of 300 instances does it fail to find the optimal solution, and the maximum deviation is less than $1 \%$. It clearly outperforms the RP, especially on instances with lower values of $\alpha$. For some instances, the RP finds a 
Table 3 Set A: Running time of the IA and RP heuristic

\begin{tabular}{|c|c|c|c|c|c|c|c|c|c|c|}
\hline \multirow[t]{2}{*}{ M } & \multirow[t]{2}{*}{$\mathrm{T}$} & \multirow[t]{2}{*}{$\alpha$} & \multicolumn{3}{|c|}{ avg time (s) } & \multicolumn{3}{|c|}{$\max$ time $(\mathrm{s})$} & \multicolumn{2}{|l|}{ IA It } \\
\hline & & & IA & $\mathrm{RP}$ & MIP & IA & $\mathrm{RP}$ & MIP & avg & $\max$ \\
\hline \multirow[t]{4}{*}{40} & \multirow[t]{4}{*}{40} & 2 & 0.013 & 9.9 & 140 & 0.016 & 11.0 & 475 & 2.91 & 9 \\
\hline & & 5 & 0.013 & 14.5 & 159 & 0.032 & 17.7 & 376 & 3.10 & 7 \\
\hline & & 8 & 0.016 & 18.0 & 170 & 0.016 & 20.9 & 519 & 3.18 & 6 \\
\hline & & 11 & 0.013 & 20.3 & 202 & 0.016 & 24.3 & 478 & 3.02 & 6 \\
\hline \multirow[t]{4}{*}{80} & \multirow[t]{4}{*}{40} & 2 & 0.017 & 47 & 571 & 0.032 & 59 & 1,673 & 2.90 & 10 \\
\hline & & 5 & 0.020 & 69 & 1,182 & 0.032 & 73 & 2,150 & 3.19 & 7 \\
\hline & & 8 & 0.020 & 92 & 994 & 0.032 & 180 & 2,114 & 3.20 & 8 \\
\hline & & 11 & 0.019 & 151 & 982 & 0.032 & 410 & 1,843 & 3.14 & 6 \\
\hline \multirow[t]{4}{*}{40} & \multirow[t]{4}{*}{80} & 2 & 0.054 & 196 & 2,907 & 0.078 & 264 & 16,734 & 3.08 & 9 \\
\hline & & 5 & 0.062 & 274 & 6,711 & 0.094 & 327 & 36,473 & 3.34 & 8 \\
\hline & & 8 & 0.063 & 286 & 4,337 & 0.093 & 1024 & 16,400 & 3.40 & 8 \\
\hline & & 11 & 0.064 & 319 & 7,357 & 0.094 & 1,303 & 43,154 & 3.36 & 8 \\
\hline
\end{tabular}

solution whose deviation from optimality is $100 \%$, which means that the RP finds a solution with profit zero while there does exist a profitable solution. Finally, we see that the RP never finds a better solution than the IA.

Table 3 presents the running times of the heuristics and CPLEX. The last two columns present the average and maximum number of iterations that the IA takes for a single starting point (i.e., for a single initial production plan). We see that the average and maximum number of iterations for the IA increases slightly as the size of the instances increase. The small number of iterations can be explained as follows. Although market selections do not satisfy a monotonicity property in general, the experiments show that it rarely happens that a market first enters and later leaves (or the other way around) a solution in the path of the IA (that is, the production plans and market selections in consecutive iterations). An intuitive explanation of this is that if only markets enter the current selection in an iteration, then demand will increase and the number of setups will increase in general. In turn, the variable cost will decrease in general and again more markets will enter the selection. By repeating this argument, markets will only enter the selection until a local optimum is found. Clearly, for a path where a market switches at most once from selected to non-selected, the number of possible market selections is limited $(\mathcal{O}(M))$ and hence the number of iterations is limited.

With respect to running time, the IA finds a solution within $0.1 \mathrm{~s}$ for all instances, while the RP takes more than $20 \mathrm{~min}$ in the worst case. Furthermore, the optimal solutions are found within $10 \mathrm{~min}$ by solving the MIP for $(M, T)=(40,40)$, but for $(M, T)=(80,40)$ and $(M, T)=(40,80)$ there are instances for which solving the MIP takes more than half an hour and almost $12 \mathrm{~h}$, respectively. Overall, we can conclude that the IA outperforms the RP both in terms of solution quality and running time for this set of instances.

Finally, if we use (10) instead of (1a) as the objective function, it turns out that both the integrality gap and the deviation from optimality are much smaller than the values 
Table 4 Set B: Performance of the IA and RP heuristic

\begin{tabular}{|c|c|c|c|c|c|c|c|c|c|c|c|c|}
\hline \multirow[t]{2}{*}{$\rho$} & \multirow[t]{2}{*}{$\alpha$} & \multirow[t]{2}{*}{ NS } & \multirow[t]{2}{*}{ LP } & \multicolumn{2}{|l|}{ IG } & \multicolumn{2}{|c|}{ avg $\operatorname{dev}(\%)$} & \multicolumn{2}{|c|}{$\max \operatorname{dev}(\%)$} & \multicolumn{2}{|c|}{ non-opt } & \multirow[t]{2}{*}{ IA-RP } \\
\hline & & & & avg & $\max$ & IA & RP & IA & $\mathrm{RP}$ & IA & $\mathrm{RP}$ & \\
\hline \multirow[t]{4}{*}{0.50} & 2 & 76 & 14 & 0.47 & 15.6 & 0.00 & 7.41 & 0.00 & 100.0 & 0 & 56 & $56-0$ \\
\hline & 5 & 81 & 5 & 0.77 & 28.1 & 0.03 & 3.48 & 3.05 & 60.9 & 1 & 52 & $52-0$ \\
\hline & 8 & 86 & 9 & 0.28 & 2.4 & 0.04 & 1.27 & 3.68 & 17.2 & 2 & 50 & $50-0$ \\
\hline & 11 & 83 & 10 & 0.22 & 2.5 & 0.00 & 2.19 & 0.30 & 48.3 & 1 & 51 & $50-0$ \\
\hline \multirow[t]{4}{*}{0.75} & 2 & 90 & 9 & 0.26 & 3.0 & 0.19 & 10.01 & 8.43 & 100.0 & 7 & 65 & $65-1$ \\
\hline & 5 & 89 & 9 & 0.27 & 6.7 & 0.09 & 5.00 & 3.27 & 100.0 & 6 & 67 & $67-1$ \\
\hline & 8 & 90 & 10 & 0.30 & 4.2 & 0.22 & 5.23 & 5.76 & 100.0 & 9 & 65 & $65-1$ \\
\hline & 11 & 91 & 15 & 0.21 & 4.9 & 0.06 & 4.14 & 3.90 & 73.7 & 3 & 58 & $57-0$ \\
\hline \multirow[t]{4}{*}{0.90} & 2 & 100 & 15 & 0.08 & 1.3 & 1.35 & 6.05 & 11.63 & 90.9 & 43 & 67 & $55-18$ \\
\hline & 5 & 100 & 20 & 0.09 & 0.8 & 1.01 & 4.78 & 12.06 & 54.5 & 30 & 66 & $59-9$ \\
\hline & 8 & 99 & 21 & 0.28 & 20.6 & 1.71 & 7.53 & 100.00 & 100.0 & 23 & 66 & $61-6$ \\
\hline & 11 & 100 & 22 & 0.10 & 1.4 & 0.58 & 5.44 & 9.57 & 72.1 & 19 & 64 & $58-4$ \\
\hline
\end{tabular}

in Table 2. The average and maximum deviation from optimality of the RP are less than $1 \%$ and $2.5 \%$, respectively, while the average and maximum deviation of the IA are even less than $0.01 \%$ and $0.1 \%$, respectively. This can be explained by the fact that $\Gamma\left(\mathcal{M}^{\prime}\right)=\sum_{m=1}^{M} R_{m}-\Pi\left(\mathcal{M}^{\prime}\right)$. This relation implies that, in the minimization problem, the deviation from optimality equals

$$
\frac{\Gamma\left(\mathcal{M}^{H}\right)-\Gamma\left(\mathcal{M}^{*}\right)}{\Gamma\left(\mathcal{M}^{*}\right)}=\frac{\Pi\left(\mathcal{M}^{*}\right)-\Pi\left(\mathcal{M}^{H}\right)}{\sum_{m=1}^{M} R_{m}-\Pi\left(\mathcal{M}^{*}\right)} \leq \frac{\Pi\left(\mathcal{M}^{*}\right)}{\sum_{m=1}^{M} R_{m}-\Pi\left(\mathcal{M}^{*}\right)}
$$

where $\mathcal{M}^{H}$ is the set of markets selected by some heuristic that considers the empty set as a candidate solution (hence $\Pi\left(\mathcal{M}^{H}\right) \geq 0$ ). As (in general) $\sum_{m=1}^{M} R_{m}$ is large relative to $\Pi\left(\mathcal{M}^{*}\right)$, the deviation from optimality will be small for the minimization problem. For example, the instances in the $\mathcal{N} \mathcal{P}$-completeness proof satisfy $\Pi\left(\mathcal{M}^{*}\right) \leq \frac{1}{2}$ and $\sum_{m=1}^{M} R_{m}=\frac{3}{2} T+\frac{1}{2}$. Hence, the deviation from optimality is smaller than $\frac{1}{3 T}$ and tends to zero as $T \rightarrow \infty$.

\subsection{Problem set B: Sparse demand matrix}

This set of instances has the property that, for each market, there is a high fraction of periods with zero demand, motivated by the fact that the $\mathcal{N} \mathcal{P}$-completeness proof of the MSP is based on instances with a sparse demand matrix. We obtain Set B from Set A by replacing a market's demand in a period by zero with some probability $\rho$, and with probability $1-\rho$ it is generated from $U[0,2 \bar{d}]$. We generated 100 instances with the cost parameters as in Set $\mathrm{A},(M, T)=(40,40)$, and $\rho \in\{0.5,0.75,0.9\}$. The results of the tests can be found in Tables 4 and 5 . 
Table 5 Set B: Running time of the IA and RP heuristic

\begin{tabular}{|c|c|c|c|c|c|c|c|c|c|}
\hline \multirow[t]{2}{*}{$\rho$} & \multirow[t]{2}{*}{$\alpha$} & \multicolumn{3}{|c|}{ avg time (s) } & \multicolumn{3}{|c|}{ max time (s) } & \multicolumn{2}{|l|}{ IA It } \\
\hline & & IA & $\mathrm{RP}$ & MIP & IA & RP & MIP & avg & $\max$ \\
\hline \multirow[t]{4}{*}{0.50} & 2 & 0.04 & 2.66 & 19.42 & 0.09 & 3.55 & 124 & 3.34 & 10 \\
\hline & 5 & 0.04 & 3.61 & 32.71 & 0.06 & 5.17 & 113 & 3.32 & 10 \\
\hline & 8 & 0.04 & 3.85 & 28.25 & 0.06 & 5.52 & 90 & 3.35 & 8 \\
\hline & 11 & 0.04 & 4.17 & 29.03 & 0.08 & 5.42 & 111 & 3.33 & 8 \\
\hline \multirow[t]{4}{*}{0.75} & 2 & 0.06 & 0.93 & 5.62 & 0.13 & 1.17 & 11.6 & 3.17 & 10 \\
\hline & 5 & 0.06 & 1.09 & 7.66 & 0.08 & 1.55 & 33.8 & 3.21 & 10 \\
\hline & 8 & 0.06 & 1.17 & 7.63 & 0.09 & 1.67 & 25.5 & 3.17 & 9 \\
\hline & 11 & 0.06 & 1.17 & 6.45 & 0.11 & 1.67 & 24.0 & 3.07 & 8 \\
\hline \multirow[t]{4}{*}{0.90} & 2 & 0.08 & 0.48 & 1.22 & 0.14 & 0.59 & 3.08 & 2.71 & 8 \\
\hline & 5 & 0.08 & 0.50 & 1.35 & 0.11 & 0.64 & 3.23 & 2.80 & 8 \\
\hline & 8 & 0.08 & 0.51 & 1.35 & 0.14 & 0.63 & 3.39 & 2.77 & 7 \\
\hline & 11 & 0.08 & 0.52 & 1.37 & 0.11 & 0.63 & 3.67 & 2.72 & 8 \\
\hline
\end{tabular}

First, we see that the IA still performs quite well in terms of average deviation from optimality. However, there is a single instance (with $\rho=0.9$ and $\alpha=8$ ) for which the IA cannot find a profitable solution while one exists. Furthermore, we see that the performance of the IA worsens as $\rho$ increases, both in terms of deviation from optimality and in terms of number of non-optimal solutions. The RP shows a decrease in performance as $\rho$ increases from 0.5 to 0.75 , but there is no clear difference between the cases $\rho=0.75$ and $\rho=0.9$. Finally, we see that for most cases the IA finds better solutions than the RP, but for $\rho=0.9$ there is also a significant number of instances for which the RP finds a better solution.

Table 5 shows that the running time of the RP and the time to solve the MIP drop dramatically as $\rho$ increases. This can be explained by the fact that, if $d_{t}^{m}=0$, then $x_{i, t}^{m}=0$ for $i=1, \ldots, t$, which means that the number of variables and constraints in the MIP decreases considerably as $\rho$ increases. Although neither heuristic performs very well for instances with high values of $\rho$, the good news is that these instances can be solved to optimality by CPLEX. For example, it takes only a couple of seconds to solve an instance with $\rho=0.9$.

\section{Summary and concluding remarks}

We studied a class of production planning problems with market selection decisions and showed that, in general, the class of problems is $\mathcal{N} \mathcal{P}$-hard and, in addition, that it is highly unlikely that there exists a polynomial-time algorithm with a constant worst-case guarantee. We provide polynomial-time solution approaches for several special cases that may occur in practice. In addition, we introduce a heuristic for solving the problem and perform extensive computational tests to compare its performance with a rounding procedure from the literature and with a commercial MIP 
solver. These results show that our heuristic finds near-optimal solution extremely fast, except when the demand patterns are unrealistically small.

Our future research will focus on identifying classes of problem instances that are polynomially solvable, as well as the development of models and algorithms for problems in which production planning is integrated with market selection and pricing.

Acknowledgments We would like to thank Retsef Levi for pointing out that the case with orders spanning up to $k$ consecutive periods (Sect. 4.4) can be solved in polynomial time, which generalizes our original result for $k=2$. The work was done while W. van den Heuvel was a visiting scholar at the Department of Industrial and Systems Engineering at the University of Florida. He would like to thank the faculty, students, and staff for their kind hospitality. His work was supported by the Netherlands Organization for Scientific Research (NWO). The work of J. Geunes and H.E. Romeijn was supported by the National Science Foundation under Grants No. DMI-0355533 and CMMI-0926508/0927930. The work of T.C. Sharkey was supported by a Graduate Research Fellowship of the National Science Foundation.

Open Access This article is distributed under the terms of the Creative Commons Attribution Noncommercial License which permits any noncommercial use, distribution, and reproduction in any medium, provided the original author(s) and source are credited.

\section{Appendix}

Theorem 3 The MSP with market-specific prices can be solved in $\mathcal{O}\left(M^{2}(\log M+\right.$ $T \log T)$ ) time.

Proof We will employ the similarities between the $M$ problems $\left(\mathrm{P}_{P}(k)\right)(k=$ $1, \ldots, M)$ that need to be solved to specialize the approach of [23]. First, note that all extreme points of the continuous relaxation of the feasible region of each of these problems are integral, and applying the approach of [23] to the relaxation of $\left(\mathrm{P}_{P}(k)\right)$ for some fixed $k$ yields an integral solution. Therefore, we can, without loss of optimality, relax the integrality constraints; we will refer to the relaxed problems as $\left(\mathrm{R}_{P}(k)\right)$. Sharkey et al. [23] construct a collection of candidate solutions indexed by

$$
\Delta=\left\{(i, j): i<j \text { and } w_{i} \neq w_{j}\right\}
$$

and denoted by $x^{(i, j)}((i, j) \in \Delta)$ that is guaranteed to contain an optimal solution to $\left(\mathrm{R}_{P}(k)\right)$. In particular, for each $(i, j) \in \Delta$, they use the complementary slackness conditions of a linear program associated with $\left(\mathrm{R}_{P}(k)\right)$ to first construct partial solutions as follows. Denote the solution to the system

$$
\begin{aligned}
& \lambda+\gamma w_{i}=r_{i} \\
& \lambda+\gamma w_{j}=r_{j}
\end{aligned}
$$

by

$$
\gamma^{(i, j)}=\frac{r_{i}-r_{j}}{w_{i}-w_{j}} \quad \text { and } \quad \lambda^{(i, j)}=\frac{r_{j} w_{i}-r_{i} w_{j}}{w_{i}-w_{j}}
$$


Then a corresponding partial solution is given by

$$
\begin{aligned}
& x_{\ell}^{(i, j)}=0 \text { if } r_{\ell}<\lambda^{(i, j)}+\gamma^{(i, j)} w_{\ell} \\
& x_{\ell}^{(i, j)}=1 \text { if } r_{\ell}>\lambda^{(i, j)}+\gamma^{(i, j)} w_{\ell} .
\end{aligned}
$$

Finally, set

$$
\begin{aligned}
I_{0}^{(i, j)} & =\left\{\ell: x_{\ell}^{(i, j)}=0\right\} \\
I^{(i, j)} & =\left\{\ell: r_{\ell}=\lambda^{(i, j)}+\gamma^{(i, j)} w_{\ell}\right\} \\
I_{1}^{(i, j)} & =\left\{\ell: x_{\ell}^{(i, j)}=1\right\} .
\end{aligned}
$$

Interestingly, these partial candidate solutions are independent of the value of $k$, and (as is shown in [23]), the time required to determine the sets $I_{0}^{(i, j)}, I^{(i, j)}$, and $I_{1}^{(i, j)}$ for all $(i, j) \in \Delta$ is $O\left(M^{2} \log M\right)$.

It now remains to complete the candidate solutions for each value of $k$ by determining the values of the variables in $I^{(i, j)}$; we will refer to the corresponding solutions as $x^{(i, j)}(k)$. These c

$$
\begin{aligned}
& \left(\mathrm{SP}_{P}(k)\right) \max \sum_{\ell \in I_{1}^{(i, j)}} r_{\ell}+\sum_{\ell \in I^{(i, j)}} r_{\ell} x_{\ell}-C_{P}\left(k, \sum_{\ell \in I_{1}^{(i, j)}} w_{\ell}+\sum_{\ell \in I^{(i, j)}} w_{\ell} x_{\ell}\right) \\
& \text { s.t. } \quad \sum_{\ell \in I^{(i, j)}} x_{\ell}=k-\left|I_{1}^{(i, j)}\right| \\
& 0 \leq x_{\ell} \leq 1 \quad \ell \in I^{(i, j)} \text {. }
\end{aligned}
$$

It is shown in [23] that $\left(\operatorname{SP}^{(i, j)}(k)\right)$ can be solved by first solving two linear knapsack problems, namely,

$$
\begin{array}{cl}
(\mathrm{KP}(k)) \max (\text { or } \min ) & \sum_{\ell \in I^{(i, j)}} w_{\ell} x_{\ell} \\
\text { s.t. } & \sum_{\ell \in I^{(i, j)}} x_{\ell}=k-\left|I_{1}^{(i, j)}\right| \\
& 0 \leq x_{\ell} \leq 1 \quad \ell \in I^{(i, j)}
\end{array}
$$

and selecting the solution of these two problems with the best objective function value to $\left(\mathrm{SP}_{P}(k)\right)$.

It is important to note that $\left(\operatorname{SP}^{(i, j)}(k)\right)$ only has a feasible solution, and thus only needs to be considered, for $\left|I_{1}^{(i, j)}\right| \leq k \leq\left|I_{1}^{(i, j)}\right|+\left|I^{(i, j)}\right|$. We will proceed by first adapting the algorithm from [23] by, for each $(i, j) \in \Delta$, solving for all relevant solutions $x^{(i, j)}(k)$ consecutively, i.e., for $k=I_{1}^{(i, j)}|, \ldots,| I_{1}^{(i, j)}|+| I^{(i, j)} \mid$. Suppose now that we have found a solution for some value $\left|I_{1}^{(i, j)}\right| \leq k<\left|I_{1}^{(i, j)}\right|+\left|I^{(i, j)}\right|$ and consider the value $k+1$. Assuming that we have recorded the sorting of the variables in the linear knapsack problems $(\mathrm{KP}(k))$ that led to their optimal solutions, then we need to 
only add in the next highest (or next lowest) value in the sorting in order to determine the solutions to $(\operatorname{KP}(k+1))$. We therefore conclude that, for a fixed $(i, j) \in \Delta$, we can solve for all candidate solutions $x^{(i, j)}(k)$ in $\mathcal{O}\left(\left|I^{(i, j)}\right| \log \left|I^{(i, j)}\right|+\left|I^{(i, j)}\right| T \log T\right)$ time, which follows from the fact that (i) we need to sort the variables in $I^{(i, j)}$ to solve all problems of the form $(\operatorname{KP}(k))$, and (ii) we need to evaluate the objective function value to $\left(\operatorname{SP}_{P}(k)\right)$ for $\mathcal{O}\left(\left|I^{(i, j)}\right|\right)$ solutions, each of which requires the solution of a lot-sizing problem.

Typically, we can expect to have $\left|I^{(i, j)}\right|=2$ for all $(i, j) \in \Delta$. If this is the case, we obtain the desired result immediately from the fact that $|\Delta|=\mathcal{O}\left(M^{2}\right)$. If, however, we may have $\left|I^{(i, j)}\right|>2$ the situation may be more complex. In general, let us define the sets $\Delta_{i}=\{j:(i, j) \in \Delta\}$ for $i=1, \ldots, M$. Now if, for a given $i$, no variable occurs in $I^{(i, j)}$ for more than one $j \in \Delta_{i}$ we have that

$$
\mathcal{O}\left(\sum_{j \in \Delta_{i}}\left|I^{(i, j)}\right|\right)=\mathcal{O}(M)
$$

so that the desired result would follow again. Finally, [23] show that, for a given $i$, the only variables that can occur in more than one of the sets $I^{(i, j)}$ are the ones for which the revenue/price pair is identical to that of market $i$. Denoting, for each $i$, the set of such markets by $D_{i}$, we obtain

$$
\sum_{j \in \Delta_{i}}\left|I^{(i, j)}\right|=\mathcal{O}\left(M+M\left|D_{i}\right|\right)
$$

and, since it is easy to see that the sets $D_{i}$ are disjoint, we obtain the desired result:

$$
\begin{aligned}
& \mathcal{O}\left(\sum_{i=1}^{M} \sum_{j \in \Delta_{i}}\left(\left|I^{(i, j)}\right| \log \left|I^{(i, j)}\right|+\left|I^{(i, j)}\right| T \log T\right)\right) \\
& =\mathcal{O}\left((\log M+T \log T) \sum_{i=1}^{M} \sum_{j \in \Delta_{i}}\left|I^{(i, j)}\right|\right) \\
& =\mathcal{O}\left(M^{2}(\log M+T \log T)\right) .
\end{aligned}
$$

\section{References}

1. Aggarwal, A., Park, J.K.: Improved algorithms for economic lot-size problems. Oper. Res. 14, 549-571 (1993)

2. Biller, S., Chan, L.M.A., Simchi-Levi, D., Swann, J.: Dynamic pricing and the direct-to-customer model in the automotive industry. Electron. Commer. J. (Dynamic Pricing Special Issue) 5(2), 309-334 (2005)

3. Chan, L.M.A., Simchi-Levi, D., Swann, J.: Pricing, production, and inventory strategies for manufacturing with stochastic demand and discretionary sales. Manuf. Serv. Oper. Manag. 8(2), 149-168 (2006) 
4. Chen, X., Simchi-Levi, D.: Coordinating inventory control and pricing strategies with random demand and fixed ordering cost: The finite horizon case. Oper. Res. 52(6), 887-896 (2004)

5. Chen, X., Simchi-Levi, D.: Coordinating inventory control and pricing strategies with random demand and fixed ordering cost: The infinite horizon case. Math. Oper. Res. 29(3), 698-723 (2004)

6. Chen, X., Zhang, J.: Duality approaches to economic lot-sizing games. Technical Report OM-2006-01, Stern School of Business, New York University (2007)

7. Deng, S., Yano, C.A.: Joint production and pricing decisions with setup costs and capacity constraints. Manag. Sci. 52(5), 741-756 (2006)

8. Drechsel, J., Kimms, A.: Computing core cost allocations for cooperative procurement. Technical report, Mercator School of Management, University of Duisburg-Essen (2007)

9. Ettl, M., Huang, P., Sourirajan, K., Ervolina, T.R., Lin, G.Y.: Supply and demand synchronization in assemble-to-order supply chains. Technical report, IBM Research Report \#RC23923, 28 March (2006)

10. Federgruen, A., Tzur, M.: A simple forward algorithm to solve general dynamic lot sizing models with $n$ periods in $\mathcal{O}(n \log n)$ or $\mathcal{O}(n)$ time. Manag. Sci. 37, 909-925 (1991)

11. Florian, M., Klein, M.: Deterministic procurement planning with concave costs and capacity constraints. Manag. Sci. 26, 669-679 (1971)

12. Garey, M.R., Johnson, D.S.: Computers and Intractability: A Guide to the Theory of NP-Completeness. W.H. Freeman and Company, New York (1979)

13. Geunes, J., Romeijn, H.E., Taaffe, K.: Requirements planning with pricing and order selection flexibility. Oper. Res. 54(2), 394-401 (2006)

14. Geunes, J., Taaffe, K., Romeijn, H.E.: Models for integrated production planning and order selection. In: Proceedings of the 2002 Industrial Engineering Research Conference (IERC). Orlando (2002)

15. Gilbert, S.M.: Coordination of pricing and multiple-period production for constant priced goods. Eur. J. Oper. Res. 114, 330-337 (1999)

16. Gilbert, S.M.: Coordination of pricing and multiple-period production across multiple constant priced goods. Manag. Sci. 46, 1602-1616 (2000)

17. Hochbaum, D.S.: Selection, provisioning, shared fixed costs, maximum closure, and implications on algorithmic methods today. Manag. Sci. 50(6), 709-723 (2004)

18. Huang, W., Romeijn, H.E., Geunes, J.: The continuous-time single-sourcing problem with capacity expansion opportunities. Nav. Res. Logist. 52, 193-211 (2005)

19. Krarup, J., Bilde, O.: Plant location, set covering and economic lot size: an $O(m n)$-algorithm for structured problems. In: Collatz, L., Wetterling, W. (eds.) Numerische Methoden bei Optimierungsaufgaben, Band 3 (Optimierung bei graphentheoretischen and ganzzahligen Problemen), volume 36 of International Series of Numerical Mathematics, pp. 155-180. Birkhänser, Basel (1977)

20. Kunreuther, H., Schrage, L.: Joint pricing and inventory decisions for constant priced items. Manag. Sci. 19, 732-738 (1973)

21. Lapide, L.: Align your organization to manage demand. Supply Chain Strateg. Newslett. MIT Supply Chain Lab, 2(7) (2006)

22. Levi, R., Geunes, J., Romeijn, H.E., Shmoys, D.B. : Inventory and facility location models with market selection. In: Jünger, M., Kaibel, V. (eds.) IPCO 2005, LNCS 3509., pp. 111-124. Springer, Berlin (2005)

23. Sharkey, T.C., Romeijn, H.E., Geunes, J.: A class of nonlinear nonseparable continuous knapsack and multiple-choice knapsack problems. Math. Program. Ser. A 126, 69-96 (2011)

24. Talluri, K.T., van Ryzin, G.J.: The theory and practice of revenue management. Kluwer Academic Publishers, Norwell, MA (2004)

25. Tamir, A.: On the core of cost allocation games defined on location problems. Transp. Sci. 27, 81-86 (1993)

26. Thomas, J.: Price production decisions with deterministic demand. Manag. Sci. 16, 747-750 (1970)

27. Thomas, J.: Price and production decisions with random demand. Oper. Res. 22(3), 513-518 (1974)

28. van den Heuvel, W., Borm, P.E.M., Hamers, H.J.M.: Economic lot-sizing games. Eur. J. Oper. Res. 176(2), 1117-1130 (2007)

29. van den Heuvel, W., Wagelmans, A.P.M.: A polynomial time algorithm for a deterministic joint pricing and inventory model. Eur. J. Oper. Res. 170(2), 463-480 (2006)

30. Wagelmans, A.P.M., van Hoesel, C.P.M., Kolen, A.: Economic lot sizing: an $\mathcal{O}(n \log n)$ algorithm that runs in linear time in the Wagner-Whitin case. Oper. Res. 40, S145-S156 (1992)

31. Wagner, H.M., Whitin, T.M.: Dynamic problems in the theory of the firm. Nav. Res. Logist. Quart. 1(5), 53-74 (1958) 
32. Wagner, H.M., Whitin, T.M.: Dynamic version of the economic lot size model. Manag. Sci. 5, 89-96 (1958)

33. Yano, C.A., Gilbert, S.M.: Coordinated pricing and production/procurement decisions: a review. In: Chakravarty, A.K., Eliashberg, J. (eds.) Managing Business Interfaces, volume 16 of International Series in Quantitative Marketing, chapter 3., pp. 65-103. Springer, US (2004) 